\title{
APPLICATION OF IMAGE PROCESSING TO EDUCATIONAL PHYSICS EXPERIMENTS AND INTERDISCIPLINARY STUDENT MOTIVATION
}

\author{
Eugenijus Macerauskas \\ Vilnius Kolegija / University of Applied Sciences, Lithuania \\ Andzej Lucun \\ Vilnius Kolegija / University of Applied Sciences, Lithuania \\ Romanas Tumasonis \\ Vilnius Kolegija / University of Applied Sciences, Lithuania \\ Aliona Kirdeikiené \\ Vilnius Kolegija / University of Applied Sciences, Lithuania \\ Antoni Kozic \\ Vilnius Kolegija / University of Applied Sciences, Lithuania

\section{Slavomir Cetyrkovski} \\ Vilnius Kolegija / University of Applied Sciences, Lithuania
}

\begin{abstract}
The paper presents an automated educational system for fast physics experiments using image processing technologies. The article shows how image processing techniques help students perform better in physical physics experiments and obtain more accurate results compared to traditional methods. The experimental system used image processing technologies, programming in LabVIEW View, automated data recording, and image processing of experimental results using MATLAB. The experimental system demonstrates students' professional specializations such as mechatronics, mathematics, and programming. The developed system allowed to improve the precision of mechanics experiments reduced the time needed to experiment and allowed automatic processing of the data accounting. The experimental physics laboratory system has already attracted specific student interest and, according to the author practice, has increased student motivation as students see realistic prospects for their future abilities. The system was created by higher education students, and includes physics, image processing technologies, not only the knowledge of electronic engineering specialization but also programming knowledge. Students of the final courses designed the experimental system, and the physics course experiments are carried out in the first course, showing collaboration between different professions and different course students, knowledge adaptability, and interdisciplinary ties.
\end{abstract}

Keywords: Image processing, Interspecialty relationship, LabVIEW-MATLAB programming. 
Macerauskas et al., 2020. Application of Image Processing to Educational Physics Experiments and Interdisciplinary Student Motivation

\section{Introduction}

Image processing applications become increasingly influential in physics education because the visual display can make traditional lessons more intriguing and accessible for students. Physics learning by Image processing applications has, therefore, been continuously improved in terms of hardware, software as well as contents and suggested that high precision measurements can be achieved by the video analysis when possible sources of errors from projections, lens distortion and marker positions were controlled.

These technologies are also relevant in the learning process, combining basic and specialty subjects (Debnath \& Sukumar, 2005). The image processing technique applied by the practice in applied physics subjects in the first years of learning. Interdisciplinary relationships are realized by combining the first-year core subjects (mathematics, physics) with the subjects taught in the later courses. The motivation for interdisciplinary subjects realized through integrated lectures and practical experiments.

The article presents a system of laboratory experiments in physics implementing five different subjects of specialty, as shown in Figure 1.

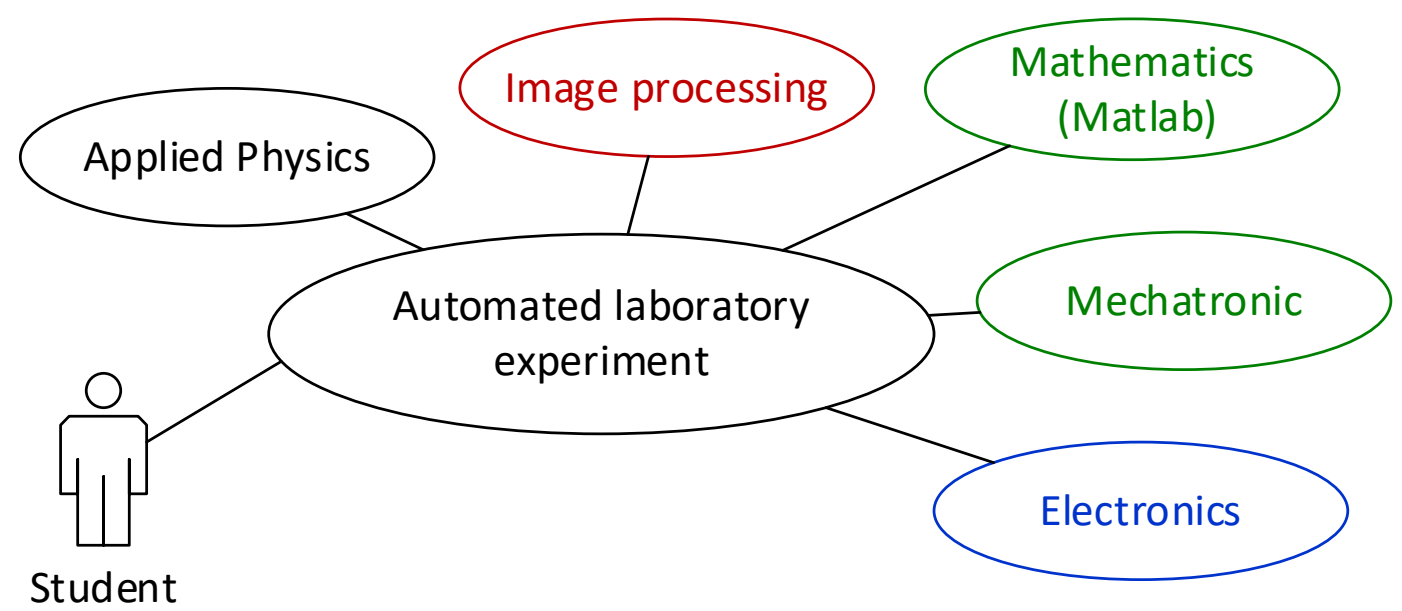

Figure 1 Interdisciplinary relationships are demonstrated through an automated experiment

In this way, during physics experiments, the student gets acquainted with the subjects of electronics, mechatronics, and informatics, which are to be thought in the following courses. 


\section{Methodology}

To illustrate the modern image processing technology in the learning process, the experiment of an applied physics subject - "Determination of the fluid Viscosity" is taken as an example.

Many methods were used to measure the viscosity of fluids. These typically based on an object moving through a fluid, in the industry, using a falling-ball viscometer. The falling ball viscometer usually measures the viscosity of Newtonian liquids and gases. The method applies Newton's law of motion under force balance on a falling ball when it reaches a terminal velocity. In Newton's law of motion for a falling ball, there exist buoyancy force, weight force, and drag force, and these three forces reach a net force of zero. The falling ball viscometer is well-suited for measuring the viscosity of a fluid, and the method has stated in international standards. The standards describe an inclined-tube method in which the tube for the falling ball inclined at 10 degrees to the vertical. Moreover, several balls used with different diameters for various dynamic viscosity measurement ranges, and a suitable ball can be selected when the fall times of the ball are not lower than the minimum fall times recorded during a testing procedure. The sample viscosity correlates with the time required by the ball to drop a specific distance, and the test results given as dynamic viscosity. The dynamic viscosity $\eta$ of a liquid is defined by the force $F$ which is required to move two parallel layers of liquid both having area $\mathrm{A}$ and separated by $d x$ with the velocity $d v$ with respect to each other (3). A spherical particle with a radius $r$ moves in a liquid under the influence of a force $F$ and the viscosity $\eta$ with a constant velocity $v$ (Stokes Law).

$$
v=\frac{F}{6 \pi \eta r}
$$

The motive force $F$ of the ball is equal to the product of the acceleration of gravity $g$ and the effective mass $m$, which can be expressed as the density difference between the ball $\rho_{1}$ and the liquid $\rho_{2}$ (Figure 2).

$$
F=m g=\frac{4}{3} \pi r^{3} g\left(\rho_{1}-\rho_{2}\right)
$$

The resistance force depends not only on the radius $r$ of the ball but also on the dimensions of the tube in which it must move. In a narrower tube, the liquid is more difficult to pass through the bead. Experimentally, it will found that the viscosity force of the ball passing through a tube of radius $R$ is greater than that of the ball passing through the tube in an unrestricted fluid, then velocity: 
Macerauskas et al., 2020. Application of Image Processing to Educational Physics Experiments and Interdisciplinary Student Motivation

$$
\eta=\frac{F}{A \frac{d v}{d x}}=\frac{2 g r^{2}\left(\rho_{1}-\rho_{2}\right)}{9 v} \frac{1}{1+2,4 \frac{r}{R}}
$$

The falling ball method has been well developed to operate this type of viscometer. The operation principle and structure of the viscometer is presented in Figure. 1.

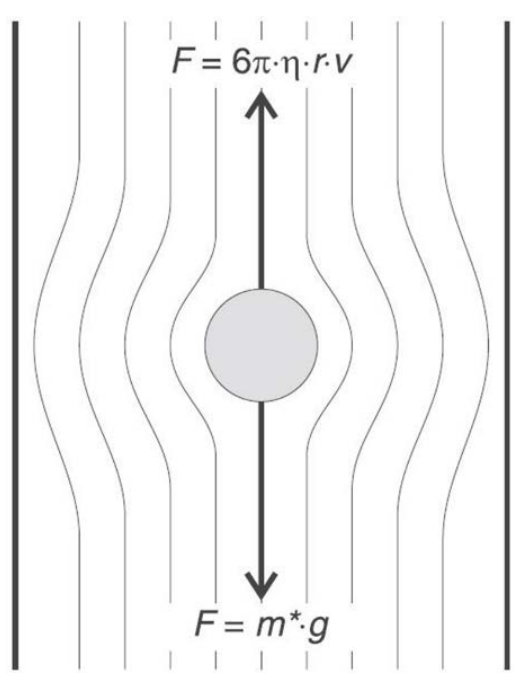

Figure 2 The forces on the falling ball in liquid

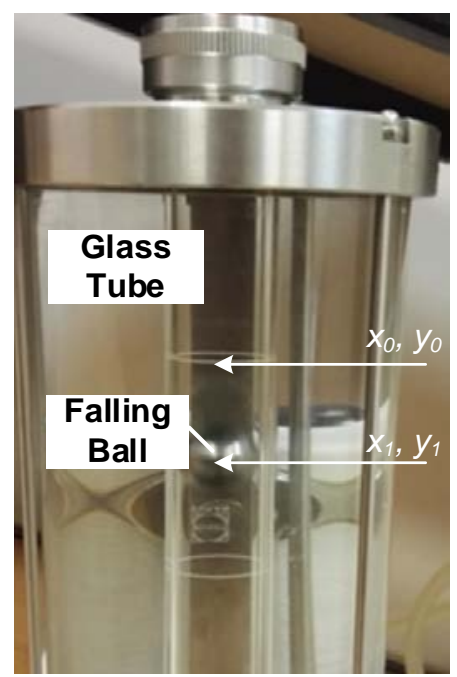

Figure 3 The structure of the viscometer with liquid

Where $x_{0}, y_{0}$ - coordinates of the starting point, $x_{1}$, $y_{1}$ - coordinates of the current point

The measuring ball moves in the glass tube, the speed of the ball depends on the viscosity of the liquid in the tube. Moreover, it is difficult to determine where the falling ball arrives at the terminal velocity, and whether the distance between the beginning record line and the starting fall position sufficient. Therefore, the purpose of this study was to develop a new method based on the traditional falling ball method, using image processing applications for describing falling ball behavior in a vertical tube.

Today's new methods of image processing in real-time systems are increasingly used in mechanical measurements. The image recognition and object tracking process are implemented using LabVIEW methods that are included in the NI Vision software. The newly created part in the software is the mean shift algorithm, which captures the change coordinates of the center of the object in the experiment process view called mean-shift algorithm (Devipriya et al., 2017).

The mean shift algorithm for moving object tracking was initially proposed to estimate the probability density function based on the distribution of points. Then according to the mean shift algorithm modes or peaks, the density function 
is determined. It is a non-parametric method, and it tracks the object for a long time, more robust compared to other tracking algorithms. To find the new location of the object that we are going to track, we need to find a vector that can suggest the direction of the moving object. This vector is called the mean shift-vector. First, we need to draw the region of interest around the object, and get the data points, approximate location of the mean of this data. Then estimate the exact location of the mean of the data by determining the mean shift vector from the initial mean - the video stream in real-time consisting of discrete frames.

In the first frame, the tracking object is selected. If $y_{0}$ is the center of an object, then the position of pixels is $\left\{x_{i}\right\} i=1,2, . . N$, where $N$ is the number of pixels in the image. The statistical histogram distribution model of the target area is given by (Devipriya M. et al., 2017):

At the current frame, the statistical histogram distribution is given by

$$
p_{h}\left(y_{0}\right)=C_{h} \sum_{i=1}^{N_{h}} k\left(\left\|\frac{y_{0-x_{i}}}{w}\right\| 2\right) \delta\left[b\left(x_{i}\right)-h\right]
$$

At the current frame, the statistical histogram distribution is given by

$$
P\left(p_{h}\left(y_{0}\right), q_{h}\right)=\sum_{h=0}^{H-1} \sqrt{p_{h}\left(y_{0}\right) q_{h}}
$$

Then computing the Bhattacharya coefficient given by

$$
P\left(p_{h}\left(y_{1}\right), q_{h}\right)=\sum_{h=0}^{H-1} \sqrt{p_{h}\left(y_{1}\right) q_{h}}
$$

Compare the coefficients and update the candidate window.

If $\left\|y_{1}-y_{0}\right\|<\varepsilon$, iteration stops, and goes equation (5).

For experimental validation, a laboratory experiment image processing system with a video camera was used. A LabVIEW application is accomplished for optical tracking of the video motion and for acquiring the trajectory coordinates for statistics. Experimental data are analyzed, and parameters are calculated using MATLAB software (Wuni et al., 2010).

Object tracking methods obtain moving objects data, which used to calculate liquid's viscosity in a physics experiment. 
Macerauskas et al., 2020. Application of Image Processing to Educational Physics Experiments and Interdisciplinary Student Motivation

\section{Realization of educational experimental system}

The modern information technology application to computer-based experiments is conducted on similar methods. It has a similar architecture that can be considered typical for microprocessor control tools with National Instruments LabVIEW software (National Instruments Corporation, 2009). The experimental system for automated physics laboratory experiments was created.

The block diagram of the experimental system is presented in Figure 3. The system consists of image processing and data accounting module implemented in LabVIEW software, mathematical calculation module realized in MATLAB mechatronic part consisting of the trigger switch, measuring ball applications, and video camera (Giannone et al., 2011; Klinger, 2008). The mechatronics part consists of an experimental mechanical start switch, which transfers the logic signal to PC by the Arduino controller (Durfee, 2011; Organtini, 2018).

The structure of the experimental system presented in Figure 4.

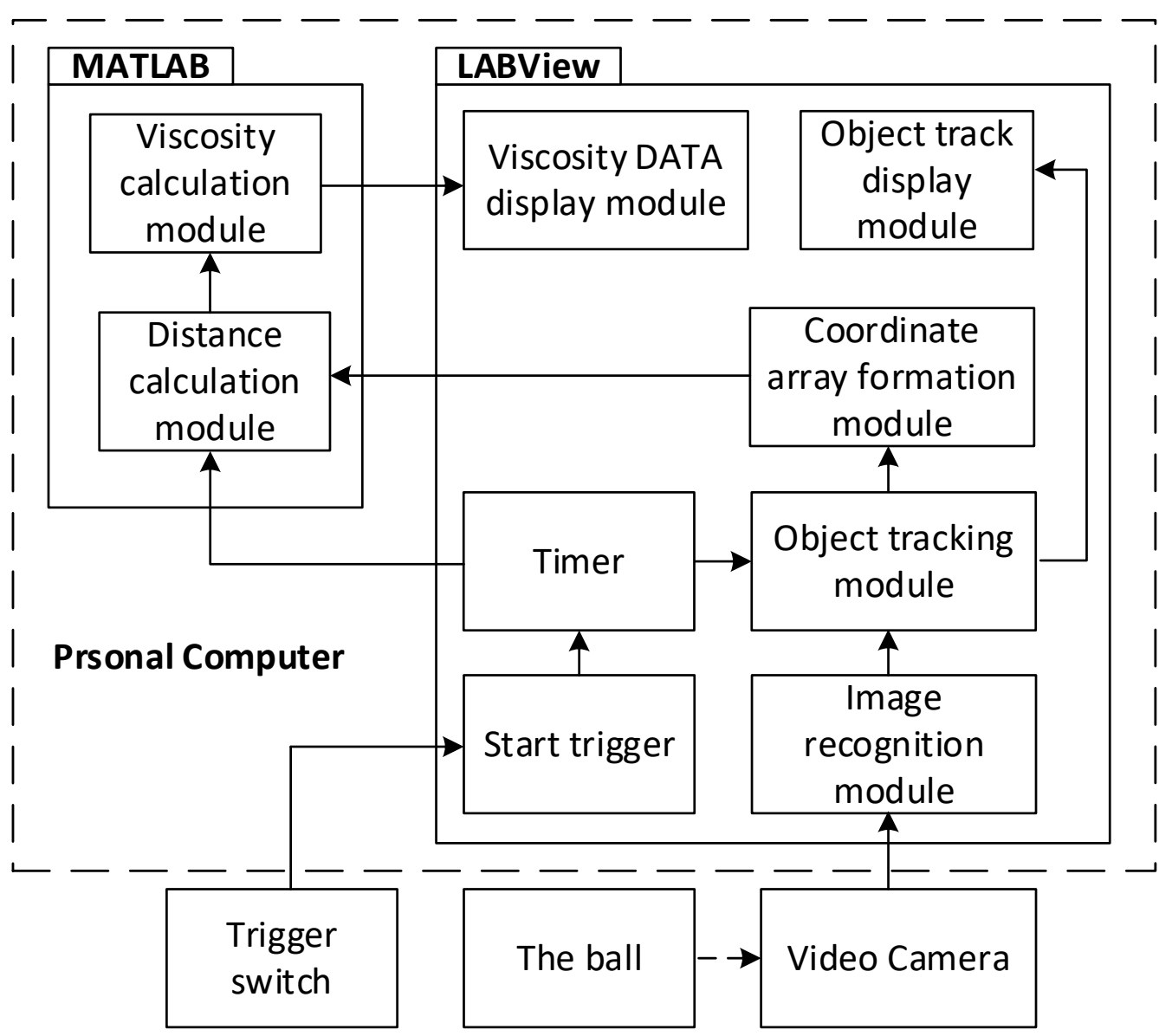

Figure 4 Block diagram for the system 
The operation of the system is illustrated in Figure 5.

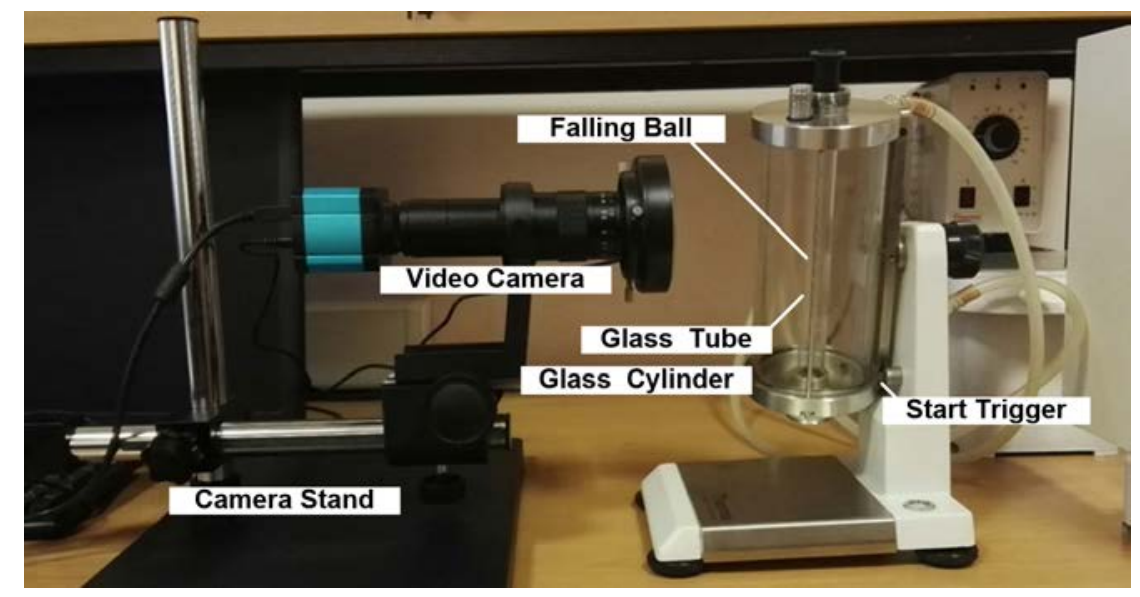

Figure 5 The system of the experiment

The main part of the system consists of a glass cylinder viscosimeter with a warm measuring ball inside (Figure 3). The video camera is mounted on the camera stand and used to record the movement of the measuring ball. From the camera, the video stream is transmitted to a personal computer, where the video stream is processed and the data applied to the mathematical calculations of the physical experiment.

Image processing is executed through the Vision Assistant module in LABView. Module configuration and processed image results are visible at each step in Figure 6.

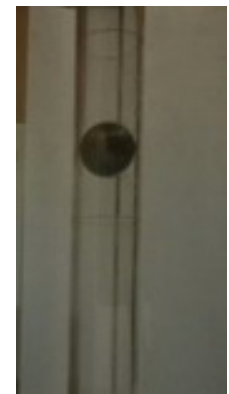

Original Image

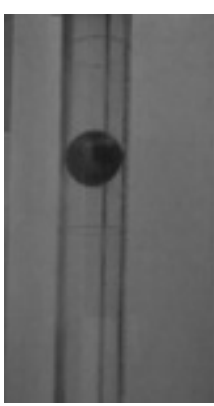

Color Plane Extraction

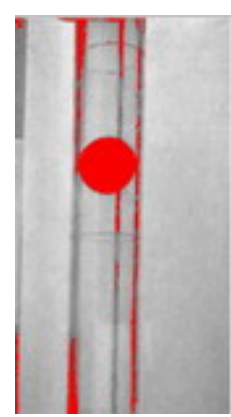

Threshold

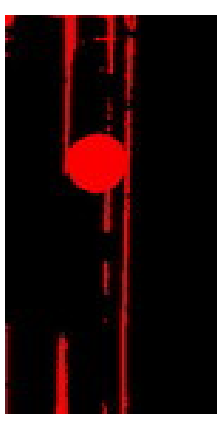

Basic Morphology

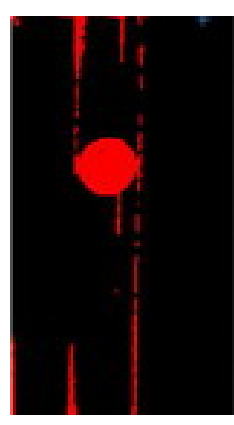

Advanced Morphology

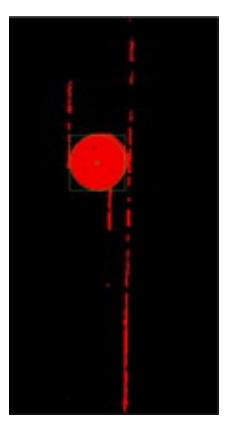

Circle Detection

Script:

Figure 6 Configuration of Vision Assistant.vi and results in image processing steps 
Macerauskas et al., 2020. Application of Image Processing to Educational Physics Experiments and Interdisciplinary Student Motivation

The activity diagram of the measuring system presented in Figure 7.

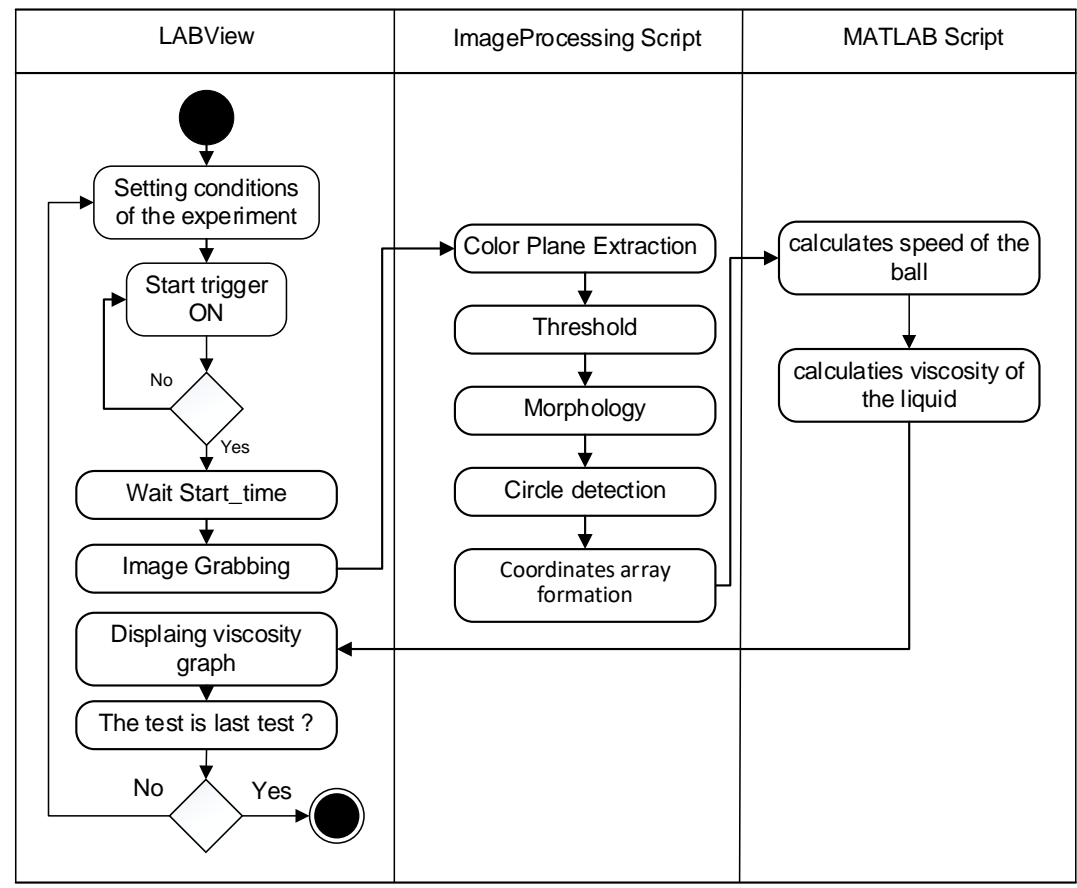

Figure 7 The activity diagram of the laboratory experiment

The experiment begins with setting the conditions for the experiment when triggered by Start Trigger pause, (Start_time) to stabilize physical processes. When the measurements start, the image is captured using the Image Grabbing function in LABView. Image processing is executed by the image processing script and consists of Colour plane extraction, Threshold, Morphology, Circle detection functions. The result of these functions is an array of coordinate data that is transfer to the MATLAB module. The MATLAB script calculates the speed of the ball, and viscosity of the liquid, as a result of the experiment if the current test is the last in the experiment LabVIEW displaying the viscosity graph of each test and of the whole experiment.

The Equipment of the Laboratory Experiments is a complex mechatronic system that contains hardware and software. Figure 6 shows the scheme of the system structure.

Experimental software developed by LabVIEW. Software running on a personal computer (National Instruments Corporation, 2009; Mandelis A., 2018).

As an illustration, in Figure 8, the measurement systems LabVIEW diagram was presented 


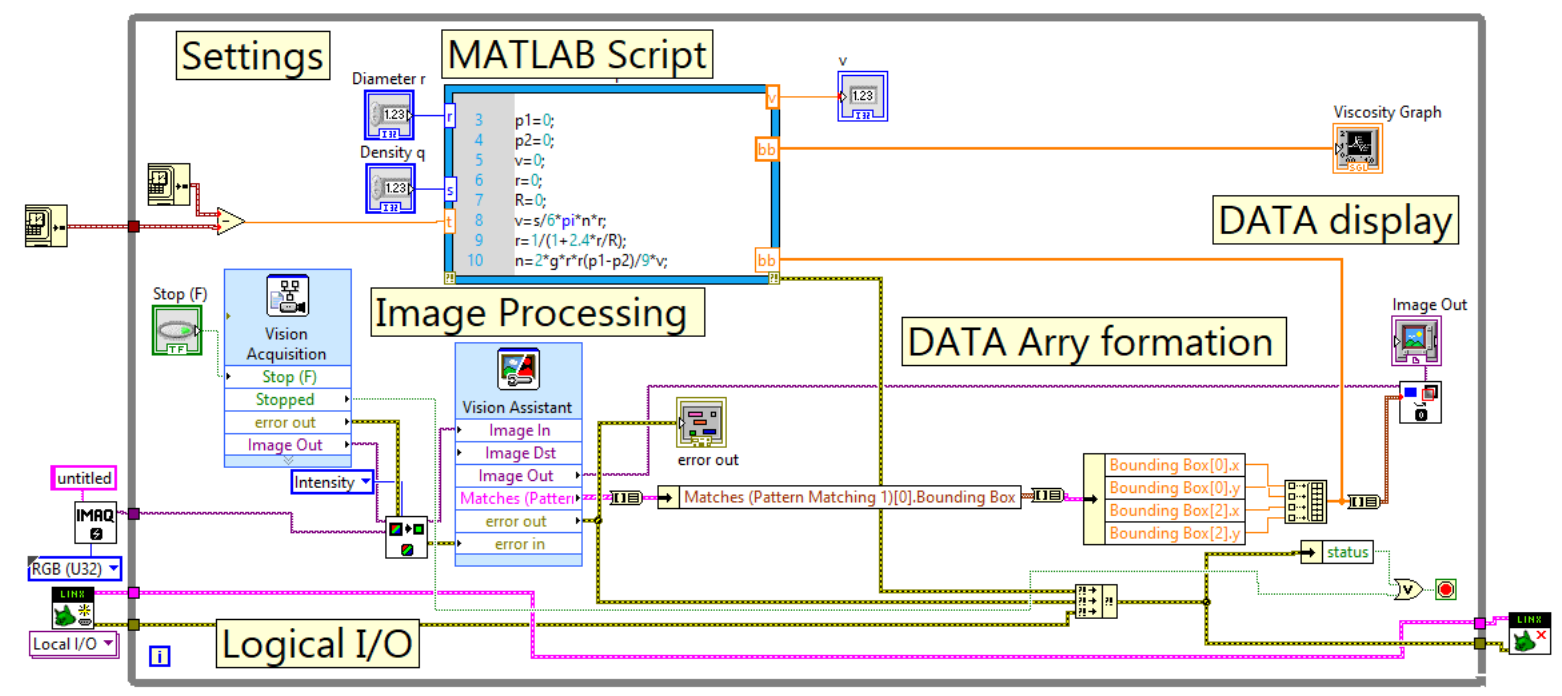

Figure 8 The LabVIEW diagram of the measurement system

The image processing part is for image capture and processing (see Figure 7). DATA array formation part designed to from a dynamic coordinate array from the image data. Logical I/O port used to receive a signal from the Start switch. MATLAB Script performs calculations based on set data by Settings part and data received from DATA array. The output of the results is executed in the data display part.

\section{Example of Physics Laboratory Experiment}

The experiment was performed with a viscometer and six balls of different diameters and density. The set of testing balls diameters $-d$, material densities $q$, speeds $-v$, and viscosity $-\eta$. The viscosity of the liquid is calculated by the formula (5).

The viscosity of distilled water at $22^{\circ} \mathrm{C}$ was measured. The data for each test were calculated automatically and displayed separately in the user interface. All data set are presented in Table 1.

Table 1 The data of the testing balls and measured parameters

\begin{tabular}{|c|c|c|c|c|}
\hline Test $\mathrm{N}_{\mathrm{o}}$ & $d, \mathrm{~mm}$ & $q, \mathrm{~kg} / \mathrm{m}^{3}$ & $v, \mathrm{~mm} / \mathrm{s}$ & $\eta, \mathrm{mPa} / \mathrm{s}$ \\
\hline 1 & 15,25 & 2340 & 3,69 & 1,12 \\
\hline 2 & 14,23 & 2340 & 5,33 & 1,11 \\
\hline 3 & 12,75 & 2340 & 6,42 & 0,89 \\
\hline 4 & 15,12 & 7800 & 1,35 & 0,95 \\
\hline 5 & 14,80 & 7800 & 2,18 & 0,99 \\
\hline 6 & 13,05 & 7800 & 3,62 & 1,12 \\
\hline
\end{tabular}


Macerauskas et al., 2020. Application of Image Processing to Educational Physics Experiments and Interdisciplinary Student Motivation

A comparison was made with the same fluid viscosity experiment without a computer-based system with image processing. Measurement accuracy data was studied, as well as the average time over which the experiment was performed. Average time spent on one test, as well as time spent on the whole experiment, and the viscosity measurement error presented in Table 2.

Table 2 Comparison of functionality data of the standard experiment system and computerbased system with image processing

\begin{tabular}{|c|c|c|c|c|c|}
\hline \multicolumn{2}{|c|}{ test $t$} & \multicolumn{2}{c|}{ exp t } & \multicolumn{2}{c|}{ m-error } \\
\hline CB S & Classic S & CB S & Classic S & CB S & Classic S \\
\hline 30s & $2 \mathrm{~min}$. & $6 \mathrm{~min}$ & $26 \mathrm{~min}$ & $12 \%$ & $23 \%$ \\
\hline
\end{tabular}

Where: - exp, $t$ - time wasted for the whole experiment, test $t$, - time spent on one test with one ball, $m$-error - error in viscosity measurements. For: $C B \_S-$ experiment with the computer-based system with image processing, Classic_S classic viscosity measurement system.

The results show that a computer-based measuring system with image processing reduces the time spent per test from $2 \mathrm{~min}$ to $30 \mathrm{~s}$. The time spent on the whole experiment, from $26 \mathrm{~min}$ to 6 is measured by taking into account the additional time that students log data manually. The most important value obtained is the measurement error, which is reduced more than twice.

It should be noted that the close viscosity values obtained when measuring with different balls could not be obtained by the classical method due to visual observation errors.

\section{Interdisciplinary relations and student motivation}

In the first year of studies students of many specialties have a practical exercise in physics. Therefore from the beginning, it is worth to cause students' motivation and interest for the sake of further studies. The author of the laboratory mentioned above experiment system is a third-year student. The Laboratory test experiment system designed by the student and implemented in the learning process demonstrates not only the interdisciplinary links between mechatronics, electronics, and programming but also encourages students to rely on their abilities. The subsequent course student develops the experimental system implemented in the physics laboratory; it motivates the students for further studies and builds their self-confidence. Experience from practical experiments with the first-year students shows that more than five interested students of Information Systems, Computer Engineering, and Electronics Engineering specialties not only 
continue to study but also show excellent results in individual subjects. Therefore, it means that the practically illustrated method of student's motivation is effective.

\section{Conclusions}

The following conclusions can be made from the analysis of the application of the automated physics laboratory experiment developed by the students:

- Image processing applications for physics laboratory experiments facilitate the experimentation process and reduces the time spent on the analysis.

- $\quad$ Computer vision techniques allow making a record of the fast processes in physics experiments, that are difficult to see for the human and also reduce the error of measurement

- $\quad$ During the first year, the student understands the practical benefits of the individual studies, and this option increases student motivation.

\section{References}

Debnath, S. (2005). College Student Motivation: An Interdisciplinary Approach to an Integrated Learning Systems Model. Journal of Behavioral \& Applied Management, 6(3), 168-188.

Devipriya, M. et al. (2017). Moving Object Tracking using FPGA. Proceedings of the International Conference on Intelligent Sustainable Systems (ICISS 2017) IEEE Xplore, 466-469.

Durfee, W. (2011). Arduino Microcontroller Guide. University of Minnesota.

Giannone, L. et al. (2011). Data acquisition and real-time bolometer tomography using LabVIEW RT. Fusion Engineering and Design, 86, I(6-8), 1129-1132.

Klinger, T. (2008). Image Processing with Lab VIEW and IMAQ Vision. Prentice Hall.

Mandelis, A. (2018). Focus on software, data acquisition, and instrumentation. Physics Today, 71(12), 58-59.

National Instruments Corporation. (2009). LabVIEW Development Guidelines, NI 2009.

Organtini, G. (2018). Arduino as a tool for physics experiments. IOP Conf. Series: Journal of Physics: Conf. Series, 1076.

Wuni, X. et al. (2010). Image processing based on seamless integration technology between LABVi LabVIEW ew and MATLAB. Proceedings of 2010 International Conference on Information, Networking and Automation (ICINA). IEEE Xplore. 